\title{
Comparison of Treatment Results and Prognostic Factors of Elderly and Young Patients Receiving Neoadjuvant Chemoradiotherapy in Rectal Cancer
}

\author{
Berrin INANC, Ozlem MERMUT \\ University of Health Sciences, Istanbul Training and Research Hospital, \\ Department of Radiation Oncology, Istanbul, TURKEY
}

\begin{abstract}
In this retrospective research, it was aimed to compared elderly ( $\leq 65$ years) and younger ( $<65$ years) rectal cancer patients who received neoadjuvant chemoradiotherapy (nCRT) and surgery. A total of 175 local advanced rectal cancer patients were included in this study. 60 elderly patients and 115 younger patients compared prognostic factors, treatment outcomes survival and toxicity between 2 groups of patients. At a median follow-up of 65 months in older patients and 80 months in younger patients. We found that Eastern Cooperative Oncology Groups (ECOG) performance status was significantly different between two groups $(p=0.001)$. None of the other treatment or patient characteristics differed significantly between the groups. The 5-year overall survival (OS) and disease-free survival (DFS) ratios were 68.0\%, 44.7\% for older patients and 79.0\%, 50.5\% for younger patients, respectively. There was no significant difference in DFS ( $p=0.311)$ between the two age groups. However, there was significant difference in $O S$ ( $p=0.05$ ) between the two age groups. ECOG of $0-I$ and $\geq 8$-week interval between nCRT and surgery were significant prognostic factors for OS and DFS in the multivariate analyses for both groups Acute haematological toxicitiy, anaemia (58\%) and thrombocytopenia (25\%) were observed more frequently in elderly patients $(p=0.005)$. There were no differences in DSF between the older and younger patients, OS rates were lower in the older patient group. A higher rate of acute haematologic toxicity was observed in older patients. However, they had similar tumour responses, treatment outcomes.
\end{abstract}

Keywords: Aged, Radiochemotherapy, Rectal neoplasms

\section{INTRODUCTION}

Rectal cancer is a complex and heterogeneous disease, especially in the locally advanced stage, which requires a multimodal treatment that includes radiotherapy, chemotherapy and surgery. Neoadjuvant chemoradiotherapy (nCRT) plays an important role in shrinking the tumour(s) to make them resectable and to conserve the rectal sphincter. Studies have compared the administration of nCRT versus postoperative chemoradiotherapy in locally advanced rectal cancer. ${ }^{1,2}$ The German Rectal Cancer Study Group ${ }^{3}$ compared nCRT and postoperative chemoradiotherapy and found that
nCRT was associated with lower local recurrence rates $(6 \%$ vs $13 \%$; $\mathrm{p}=0.06)$ and treatment-associated toxicity $(27 \%$ vs $40 \% ; \mathrm{p}=0.001)$. In the 10 year results of that study, overall survival (OS) was similar in both groups (59.9\% vs $59.6 \%$; $=0.85)$, but local recurrence was lower in the nCRT arm $(7.3 \%)$ than the postoperative chemoradiotherapy arm $(10.1 \%)$. The study also found that nCRT was associated with a reduced tumour volume and increased rate of sphincter-sparing procedures. Due to these advantages, nCRT is recommended for patients with locally advanced rectal cancer. 
Rectal cancer is usually seen in older adults over the age of 65 . However, recent data have shown a rapid decline in the incidence of rectal cancer among those ages 65 or older. ${ }^{4}$ A retrospective cohort study of the Surveillance, Epidemiology and Result program (SEER) programme found that the incidence of rectal cancer in patients younger than 50 years has been increasing. ${ }^{5}$ Another study estimated that by 2030 , the incidence of rectal cancer will increase by $124.2 \%$ for patients 20 to 34 years of age. ${ }^{6}$ The reason for this increase is unknown, but it could be due to the fact that a young adult patients are clinicopathologically and genetically different from older patients. Therefore, we aimed to investigate prognostic factors, treatment outcomes, survival and toxicity in older ( $\geq 65$ years) and younger $(<65$ years) rectal cancer patients treated with nCRT and surgery.

\section{PATIENTS AND METHOD}

\section{Eligibility Criteria}

In this retrospective study, we evaluated 175 patients who were newly diagnosed with locally advanced rectal cancer after biopsy and received nCRT between February 2011 and December 2018. The patients were divided into two groups: older $(\geq$ 65 years) and younger ( $<65$ years). The eligibility criteria were patients between the ages of 18 and 80 years diagnosed with histologically confirmed rectal cancer (clinical stage: T3, T4 or $\mathrm{N}+$ ) who had no distant metastasis, had not received palliative radiotherapy (RT) and had no prior rectal RT.

The study was approved by the Human Research Ethics Committee of Istanbul Training and Research Hospital Ethics Committee (approval number: 2020-1792) according to the Declaration of Helsinki. Informed consent was obtained from all patients after thorough explanation of the study. All related laboratory and pathology results were obtained from hospital data, and data related to treatment follow-up were obtained from clinical files.

\section{Radiotherapy and Chemoradiotherapy Data}

All patients were diagnosed by biopsy prior to receiving nCRT treatment. All patients received an external beam of RT (1.8 - 2.0 Gy daily fractions with 6/18 MV photon beams, 5 days a week) over a period of 6 weeks using linear accelerators. RT was conducted using field-in-field Intensity modulated radiotherapy (IMRT) or the four-field box three-dimensional conformal technique or VMAT arc tecnique. The clinical target volume included the gross tumour volume as well as pelvic lymph nodes and their direct extension. The planning treatment volume was the clinical target volume plus a $1 \mathrm{~cm}$ margin (for the rectum) and a $0.7 \mathrm{~cm}$ margin (for the pelvic lymph nodes). All patients received a phase 2 tumour bed boost. Pelvic lymph nodes and the rectum were subject to 40-45 Gy and tumour(s) to $50.4 \mathrm{~Gy}$.

Concurrent chemotherapy was administered from the first day of RT to the last day of RT. The chemotherapy regimen continued with a 5 -fluorouracil (5-FU) intravenous infusion $\left(180 \mathrm{mg} / \mathrm{m}^{2} /\right.$ per day) for 7 days or oral 5-FU-derived capecitabine (825 $\mathrm{mg} / \mathrm{m}^{2}$ twice a day) for 5 days of RT. After surgery, patients were referred to the medical oncology clinic for adjuvant chemotherapy.

\section{Assessment of Response and Follow-up}

Treatment toxicity was evaluated using the Common Terminology Criteria for Adverse Events version 3.0. During RT, patients were evaluated at least once a week with a clinical examination, and their blood count and biochemistry were analyzed. Treatment response was assessed by pelvic magnetic resonance imaging (MRI) and colonoscopy after 6-10 weeks of nCRT. After surgery, treatment response was evaluated by the gastrointestinal pathologist. The modified Ryan classification was used to measure tumour regression grade (TRG); $\mathrm{TRG} 0=$ pathological complete response $(\mathrm{pCR})$, TRG1 $=$ few groups of tumour cells remaining, $\mathrm{TRG} 2=$ residual tumour remaining and $\mathrm{TRG} 3=$ stable disease. $^{7}$

Subsequent controls included physical examinations and radiological imaging every 3 months. Follow-up were conducted every 3 months for the first 2 years and then every 6 months for years 3-5. During the follow-up period, MRI examination was conducted in patients with suspected local or regional recurrence. 


\section{Statistical Analysis}

A comparison of patients and treatment features between the younger and older groups were carried out using Pearson's chi-squared. Kaplan-Meier survival curves were used to analyze diseasefree survival (DFS) and overall survival (OS) of the two groups. OS was defined as the time between initiation of nCRT and the date of death/last visit. DFS was the interval between the initiation of nCRT and observation of disease progression. The Cox proportional-hazards model was used to investigate the association between patients, treatment variables and survival in univariate/multivariate analyses. All analyses were performed with a 95\% confidence interval (CI) and 5\% significance level using version 17.0 (SPSS, Inc., Chicago, IL, USA) for Windows.

\section{RESULTS}

\section{Patient Characteristics}

Retrospective data, available treatment features and survival records of 175 locally diagnosed advanced rectal cancer patients treated with nCRT were analysed. Patients were stratified according to older $(n=60,34 \%)$ and younger $(n=115,66 \%)$ age. Table 1 present some baseline characteristics of the patients and treatments. ECOG performance status was significantly different between the older and younger groups $(p=0.001)$. None of the other treatment or patient characteristics differed significantly between the groups.

\section{Tumour Response and Survival Outcomes}

Table-1 shows treatment response ratios and some pathological features. Surgery was performed after nCRT. Low-anterior resection was conducted in 43 older patients $(71.7 \%)$ and 73 younger patients $(63.5 \%)$. Abdominoperineal resection was conducted in 17 older patients (28.3\%) and 42 younger patients $(36.5 \%)(p=0.272)$. No significant differences were observed between the two groups in terms of $\mathrm{pCR}(\mathrm{p}=0.311)$ or TRG $(\mathrm{p}=0.900)$. Pathological complete response (pCR) was observed in 7 older patients $(11.7 \%)$ and 22 younger patients (19.1\%).
At a median follow-up of 65 months (range: 5377 months), 19 older patients (37\%) were exitus. At a median follow-up of 80 months (range: $72-88$ months), 24 younger patients (68.7\%) were exitus. The 5-year OS and DFS ratios were $68.0 \%$ and $44.7 \%$, respectively, for older patients and $79.0 \%$ and $50.5 \%$, respectively, for younger patients. There was no significant difference in DFS [hazard ratio $(\mathrm{HR})=1.01 ; 95 \% \mathrm{CI}: 0.820-1.625 ; \mathrm{p}=0.311]$ between the two age groups. However, there was significant difference in OS $[\mathrm{HR}=0.722 ; 95 \% \mathrm{CI}$ : $0.433-3.310 ; \mathrm{p}=0.05$ ] between the two age groups (Figure-1).

Table 2 depict the factors that were evaluated in the univariate (prediction of favourable results) and multivariate (independent prognostic factors) analyses to determine their influence on the OS and DFS outcomes. ECOG of 0-I (vs II-III), surgery time $>8$ weeks (vs $\leq 8$ weeks) and RT dose of 50 Gy (vs 45 Gy) predicted favourable OS in elderly patients and were shown to be good independent prognostic factors in this age group $(\mathrm{p}<0.005$ for each). However, in younger patients, RT dose of 50 Gy (vs 45 Gy) and ECOG of 0-I (vs II-III) were not shown to be independent prognostic factors of OS (p>0.005). ECOG of 0-I (vs II-III), surgery time $>8$ weeks (vs $\leq 8$ weeks) and oxaliplatinbased (vs 5-FU-based) adjuvant chemotherapy predicted favourable DFS and were shown to be independent prognostic factors in younger patients ( $p<0.005$ for each). However, oxaliplatin-based (vs 5-FU-based) adjuvant chemotherapy was not found to be an independent prognostic factor for older patients.

\section{Treatment Toxicity}

Table 1 presents the treatment side effects according to age group. Acute haematological toxicitiy, anemia (58\%) and thrombocytopenia (25\%) were observed more frequently in elderly patients $(\mathrm{p}=$ 0.005). In terms of non-haematological toxicity, diarrhoea and proctitis were observed in both age groups, and there were no differences in the rates of these side effects between younger and older patients $(\mathrm{p}=0.194)$. Common late complications were rectal bleeding (15.0\%), ileus (8.3\%) and fistula $(3.3 \%)$ in older patients. Grade- 3 and higher 
International Journal of Hematology and Oncology

Table 1. Pateints characterics and toxicity outcome in elderly and young patients

\begin{tabular}{|c|c|c|c|c|}
\hline Variable & Strata & Older $(n=60)$ & Younger $(n=115)$ & $P$ value \\
\hline Age, Mean $\pm S D$ (years) & & $72.7 \pm 5.30$ & $52.5 \pm 9.06$ & \\
\hline \multirow[t]{2}{*}{ Sex } & Male & 42 (70\%) & $70(\% 60.9)$ & $0.233^{a}$ \\
\hline & Female & 18 (30\%) & 45 (\%39.1) & \\
\hline \multirow[t]{2}{*}{ Localization } & Distal & $25(41.7 \%)$ & $53(46.5 \%)$ & $0.543^{a}$ \\
\hline & Other & 35 (58.2\%) & $61(53.3 \%)$ & \\
\hline \multirow[t]{2}{*}{ Histopathology } & Adenocarcinoma & 50 (83.3\%) & 104 (90.4\%) & $0.170^{a}$ \\
\hline & Other & $10(16.7 \%)$ & $11(9.6 \%)$ & \\
\hline \multirow[t]{2}{*}{ ECOG } & $0-1$ & 22 (36.6\%) & 105 (91.3\%) & $0.001^{a}$ \\
\hline & $\|-\|$ & 38 (28.3\%) & $10(8.7 \%)$ & \\
\hline \multirow[t]{2}{*}{ Clinical stage } & T3-4, NO & $6(10 \%)$ & 16 (13.9\%) & $0.459^{a}$ \\
\hline & $\mathrm{T} 3-4, \mathrm{~N}_{+}$ & 54 (90\%) & $99(86.2 \%)$ & \\
\hline \multirow{2}{*}{$\begin{array}{c}\text { Tumor regression } \\
\text { grade (TRG) }\end{array}$} & $0-1$ & $28(46.7 \%)$ & $43(37.4 \%)$ & $0.090^{\mathrm{a}}$ \\
\hline & $\|-I\|$ & 32 (53.3\%) & 72 (62.6\%) & \\
\hline \multirow[t]{2}{*}{ Surgery type } & LAR & $43(71.7 \%)$ & $73(63.5 \%)$ & $0.272^{a}$ \\
\hline & MILES operation & 17 (28.3\%) & $42(36.5 \%)$ & \\
\hline \multirow[t]{2}{*}{ Time to surgery } & $\geq 8$ week & $44(73.3 \%)$ & 85 (73.9\%) & $0.938^{a}$ \\
\hline & $<8$ week & $16(26.7 \%)$ & $30(26.1 \%)$ & \\
\hline \multirow{3}{*}{$\begin{array}{l}\text { Chemoradiotherpy } \\
\text { response }\end{array}$} & Complet response & 7 (11.7\%) & $22(19.1 \%)$ & $0.311^{\mathrm{a}}$ \\
\hline & Partial response & $40(66.7 \%)$ & $64(55.7 \%)$ & \\
\hline & Stable disease & $13(21.7 \%)$ & $26(25.27 \%)$ & \\
\hline \multirow[t]{2}{*}{ Radiotherapy doses } & $45 \mathrm{~Gy}$ & $13(22 \%)$ & $22(19.3 \%)$ & $0.671^{a}$ \\
\hline & 50.4 Gy & $46(78 \%)$ & $92(80.7 \%)$ & \\
\hline \multirow[t]{2}{*}{ Adjuvant chemoterapy } & 5-Flourourasil based & $23(38.3 \%)$ & $55(47.8 \%)$ & \\
\hline & Oxaliplatin based & $37(61.7 \%)$ & $60(52.2 \%)$ & $0.194^{a}$ \\
\hline Distant metastasis & & $10(15.3 \%)$ & $24(20.8 \%)$ & $0.125^{a}$ \\
\hline Local recurrence & & 0 & $6(0.5 \%)$ & $0.145^{\mathrm{a}}$ \\
\hline \multirow{2}{*}{$\begin{array}{l}\text { Follow-up, Median } \pm S D \text {, } \\
\text { month, (range) }\end{array}$} & $65 \pm 5.90$ & $80 \pm 4.10$ & & \\
\hline & $(53-77)$ & $(72-88)$ & & \\
\hline Exitus & 19 (37\%) & $24(68.3 \%)$ & & $0.115^{\mathrm{a}}$ \\
\hline Alive & 41 (20.9\%) & 91 (79.1\%) & & \\
\hline Toxicities & \multicolumn{2}{|c|}{ Acute hematologic complication } & & \\
\hline Anemia & Present & $35(58 \%)$ & $29(25.2 \%)$ & $0.005^{\mathrm{a}}$ \\
\hline Leucopenia & Present & $8(13 \%)$ & $15(13 \%)$ & 0.540 \\
\hline \multirow[t]{2}{*}{ Trombocytopenia } & Present & $15(25 \%)$ & $12(10.4 \%)$ & $0.678^{\mathrm{a}}$ \\
\hline & \multicolumn{2}{|c|}{ Acute-non hematologic complication } & & \\
\hline Diarrhoea & Present & $46(78 \%)$ & $92(80.7 \%)$ & $0.194^{a}$ \\
\hline \multirow[t]{2}{*}{ Proctitis } & Present & 7 (11.7\%) & $22(19.1 \%)$ & $0.722^{\mathrm{a}}$ \\
\hline & Late complication & & & \\
\hline Rectal bleeding & Present & $9(15 \%)$ & $11(9.5 \%)$ & $0.545^{\mathrm{a}}$ \\
\hline Fistula & Present & $5(8.3 \%)$ & $3(2.6 \%)$ & $0.899^{a}$ \\
\hline Any grade-3 toxicities & & $2(3.3 \%)$ & $1(0.8 \%)$ & 0.058 \\
\hline
\end{tabular}

Abbrevation: $S D=$ standart derivation, ECOG= Eastern Cooperative Oncology Group, LAR=Low-anterior Resection, MILES=Abdominoperineal resection, ${ }^{a}=$ Pearson Chi-square test 


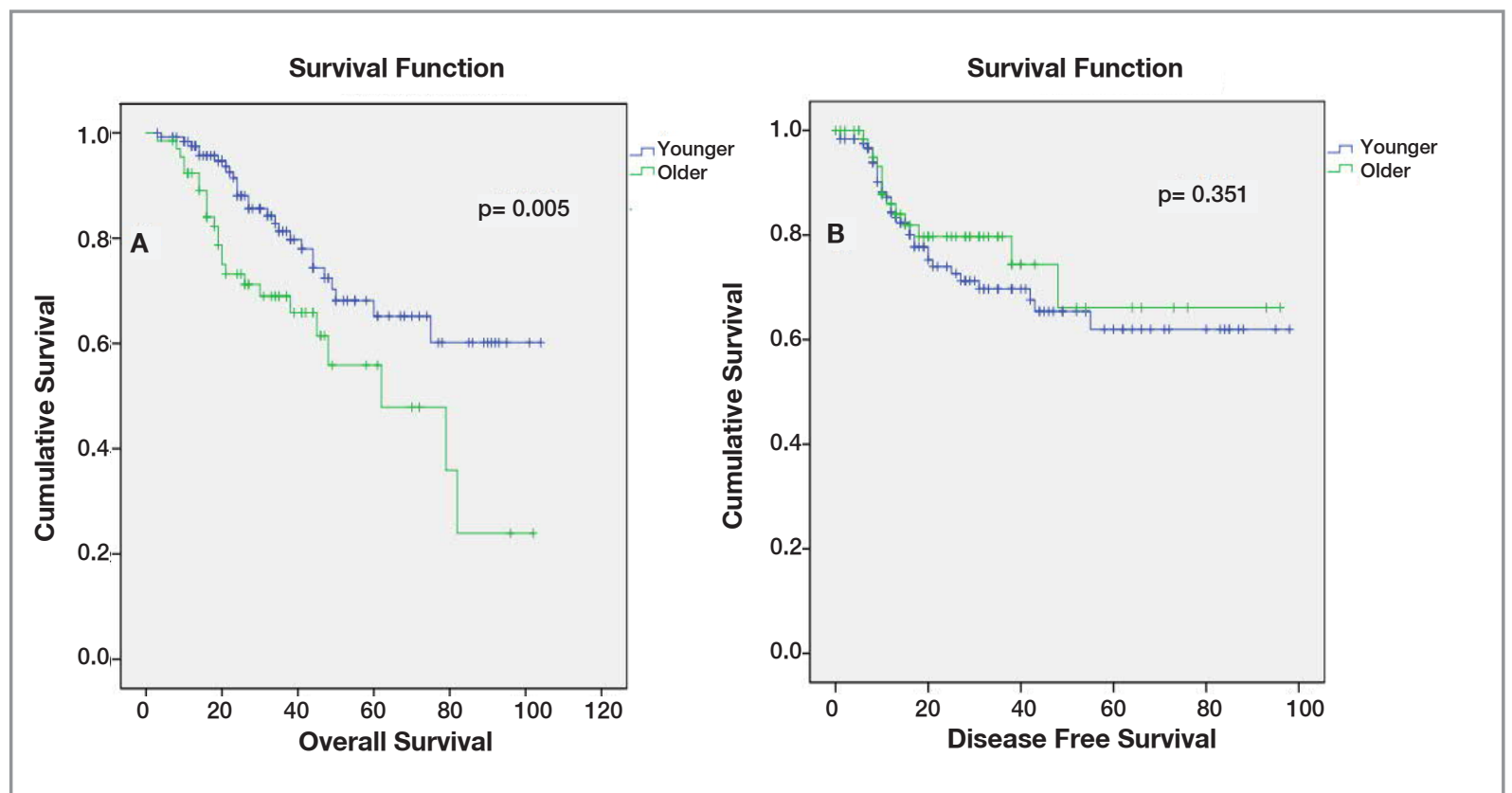

Figure 1A-B. Kaplan-Meier curves for OS and DFS

late complications occurred in two older patients (3.3\%) and one younger patient $(0.8 \%)$. No significant difference was found between the two groups in terms of late complications $(\mathrm{p}=0.058)$. No grade IV or V toxicity complications were found in either group.

\section{DISCUSSION}

More than half of rectal cancer patients are 70 years of age or older. However, in recent years, an increasing rate of rectal cancer has been observed in younger patients ( $<50$ years). With the increased incidence of rectal cancer and more aggressive in

\begin{tabular}{|c|c|c|c|c|c|c|}
\hline & & Older $(\geq 65 y$ & & Younger $(<$ & year) & \\
\hline & $\begin{array}{l}\text { Univariate } \\
\mathrm{p} \text { value }\end{array}$ & $\begin{array}{l}\text { Multivariate } \\
\mathrm{p} \text { value }\end{array}$ & $\mathrm{HR}$ & $\begin{array}{l}\text { Univariate } \\
\mathrm{p} \text { value }\end{array}$ & $\begin{array}{l}\text { Multivariate } \\
\mathrm{p} \text { value }\end{array}$ & $\mathrm{HR}$ \\
\hline & \multicolumn{6}{|c|}{ Overall survival } \\
\hline \multicolumn{7}{|l|}{ ECOG } \\
\hline (0-1vs II-III) & 0.022 & 0.007 & 1.90 & 0.963 & - & - \\
\hline \multicolumn{7}{|l|}{ Surgery time } \\
\hline ( $\geq 8$ week vs $<8$ week) & 0.005 & 0.004 & 2.67 & 0.001 & 0.001 & 2.73 \\
\hline \multicolumn{7}{|l|}{ Radiotheray doses } \\
\hline \multirow[t]{2}{*}{ (50Gy vs 45Gy) } & 0.03 & 0.08 & 2.46 & 0.780 & - & - \\
\hline & \multicolumn{6}{|c|}{ Disease-free survival } \\
\hline \multicolumn{7}{|l|}{ ECOG } \\
\hline (0-1vs II-III) & 0.024 & & 2.40 & 0.003 & 0.021 & 2.77 \\
\hline Surgery time & & & & & & \\
\hline ( $\geq 8$ week vs $<8$ week) & 0.005 & 0.004 & 2.27 & 0.001 & 0.001 & 2.73 \\
\hline \multicolumn{7}{|l|}{ Adjuvant chemoterapy } \\
\hline (5-FU vs oxlipalitin based) & 0.018 & - & - & 0.033 & 0.025 & 1.94 \\
\hline
\end{tabular}


younger patients, optimal treatment selection has become more important in both younger and older patients with locally advanced rectal cancer. Based on all these reasons and the study performed by Guillerme et al. ${ }^{8}$, we divided our patients as aged under 65 and over and investigated the factors affecting the treatment results.

The present study found no significant difference in DFS between older and younger patients. However, a significantly lower OS was observe in older patients compared to younger patients. In the study conducted by Grosso et al. ${ }^{9} 235$, over 65 years old and 211, 65 years and younger colorectal cancer patients were examined. They found that 3 -year and 5-year survival rates were worse in the elderly patient group. Similarly, 5-year survival was found to be poor in older patients in our study (79\% in younger patients vs $68 \%$ older patients). Jiang et al. ${ }^{10}$ compared 877 younger ( $<70$ years) and 295 older ( $\geq 70$ years) patients who received nCRT and curative surgery. They found no significant difference in DFS based on age in either univariate $(\mathrm{p}=$ 0.073 ) or multivariate $(\mathrm{HR}=0.93 ; 95 \% \mathrm{CI}: 0.68$, 1.26; $\mathrm{p}=0.63$ ) analyses. Moreover, young and older patients were similar OS time $(\mathrm{HR}=1.228$; 95\% CI: 0.99-1.86; $\mathrm{p}=0.20)$. In our analyses, 5 -year DFS was similar between older (44.7\%) and younger $(50.5 \%)$ patients $(\mathrm{p}=0.311)$.Additionally, the local recurrence rate $(0 \%$ vs $0.5 \%)$ and distant metastasis ( $15.3 \%$ vs $20.8 \%$ ) were similar between older and younger patients. We believe that the reason for local recurrence and distant metastasis being more common in young patients is the aggressive course of rectal cancer.

ECOG and Karnofsky score are a parameter used in rectal and other cancers to evaluate the patient's general condition and to assess patients's pretreatment status. These performance status scales are mainly used to determine whether patients are eligible for a particular treatment. Performance status has been shown to be a predictor of survival in cancer patients in many studies. ${ }^{11,12}$ ECOG status was naturally higher (ECOG II-III) in older patients due to additional chronic diseases, while it was lower in younger patients $(\mathrm{p}=0.001)$. Moreover, we found that ECOG 0-I (vs II-III) was an independent prognostic factor for OS and DFS in both groups.
In locally advanced rectal cancer, nCRT treatment is generally recommended and surgery is performed 6 weeks after treatment. Longer waiting times after nCRT resulted in better tumor shrinkage, sphincter preservation and this was associated with better survival time. As a result of the studies ${ }^{13-16}$ conducted in recent years, the period between treatment and surgery has been increased to 8 weeks or more. Moreover, published studies reported that an interval of greater than 8 weeks between nCRT and surgery was associated with improved pCR and better DFS rates. In our study, we found that an interval of greater than 8 weeks between surgery and nCRT was an independent prognostic factor for OS and DFS in both groups.

National Comprehensive Cancer Network guidelines ( NCCN) suggest a radiation dose of 45-50 Gy in 25-28 fractions for nCRT for the treatment of rectal cancer. We used 45 or 50 Gy RT in line with these guidelines. Multiple studies have demonstrated that short-course RT ( 25 Gy in 5 fractions) were as effective as long course RT (50.4 Gy in 25 fractions). ${ }^{17,18}$ These studies found no differences in sphincter preservation, local control or OS. Park and $\mathrm{Kim}^{19}$ compared three dose fractionations (50 Gy in 25 fractions, 50.4 Gy in 28 fractions and 45 Gy in 25 fractions) and found no significant difference in local control, OS or DFS. In contrast, in our study, an RT dose of 50 Gy (vs 45 Gy) was found to be an independent prognostic factor for OS in older patients.

Another important variable was the adjuvant chemotherapy regimen after surgery. The International Society of Geriatric Oncology recommends 5-FUbased adjuvant chemotherapy for elderly patients with advanced rectal cancer. ${ }^{20}$ Older patients are less likely to take oxaliplatin-based chemotherapy than younger patients. In the study performed by Sun et al. $.^{21}, 43$ patients, $\geq 70$ years, were operated on and then evaluated for adjuvant chemotherapy. Patients who received and did not receive adjuvant chemotherapy were compared. Survival advantage of adjuvant chemotherapy was not shown in the elderly patient group. It has been observed to increase toxicity. In our study, with the exception of acute hematologic toxicities in older patients, adjuvant chemotherapy did not have a significant effect on OS or DFS in older patients. However, 
oxaliplatin-based (vs 5-FU-based) adjuvant chemotherapy was found to be an independent prognostic factor for DFS in younger patients.

Although nCRT and surgical treatment (multimodal treatment) provide a survival advantage in local advanced stage rectal cancer and reduce local recurrence, treatment-related side effects are increased. Most of the time, side effects are well tolerated, but elderly patients occasionally interrupt treatment because of side effects. Acute and late toxicity rates were found to be better in our study than in previous studies. ${ }^{22,23}$ Acute haematological toxicity rate was higher in older patients. Although our late toxicity rates were not statistically significant, they were close to significant. Thus, late toxicity can concluded to be higher in older patients.

There was several limitation to our study. First, the number of patients was not homogeneously distributed. Second, we could not determine if the cause of death was due to a comorbidity.

In conclusion, although there were no differences in DSF between the older and younger patients, OS rates were lower in the older patient group. ECOG of $0-\mathrm{I}$ and $>8$-week interval between nCRT and surgery were significant prognostic factors for OS and DFS in the multivariate analyses for both groups. A higher rate of acute haematologic toxicity was observed in older patients than younger patients. However, they had similar tumour responses, treatment outcomes and survival. The findings of this study will help with the development of treatment strategies in older and younger patients receiving nCRT for rectal cancer.

\section{REFERENCES}

1. Sauer R, Becker H, Hohenberger W, et al. Preoperative versus postoperative chemoradiotherapy for rectal cancer. N Engl J Med 351: 1731-1740, 2004.

2. Wagman R, Minsky BD, Cohen AM, et al. Sphincter preservation in rectal cancer with preoperative radiation therapy and coloanal anastomosis: long term follow-up. Int J Radiat Oncol Biol Phys 42: 51-7, 1998.

3. Sauer R, Liersch T, Merkel S, et al. Preoperative versus postoperative chemoradiotherapy for locally advanced rectal cancer results of the German CAO/ARO/AIO-94 randomized phase III trial after a median follow-up of 11 years. J Clin Oncol 30: 1926-33, 2012.
4. Siegel RL, Miller KD, Goding Sauer A, et al. Colorectal cancer statistics. CA Cancer J Clin 70: 145-164, 2020.

5. Bailey CE, Hu CY, You YN, et al. Increasing disparities in the age-related incidences of colon and rectal cancers in the United States, 1975-200. JAMA Surg 150: 17-22, 2015.

6. Weinberg BA, Marshall JL, Salem ME. The growing challenge of young adults with colorectal cancer. Oncology (Williston Park) 31: 381-389, 2017.

7. Ryan R, Gibbons D, Hyland JM, et al. Pathological response following long-course neoadjuvant chemoradiotherapy for locally advanced rectal cancer. Histopathology 47: 141-146, 2005.

8. Guillerme F, Clavier JB, Nehme-Schuster H, et al. Age impacts the pattern care for elderly patients with rectal cancer. Int J Colorectal Dis 29: 157-163, 2014.

9. Grosso G, Biondi A, Marventano S, et al. Major postoperative complications and survival for colon cancer elderly patients. BMC Surg 12(Suppl 1): 1-20, 2012.

10. Jiang DM, Raissouni S, Mercer J, et al.Clinical outcomes of elderly patients receiving neoadjuvant chemoradiation for locally advanced rectal cancer. Ann Oncol 26: 2102-2106, 2015.

11. Extermann M, Overcash J, Lyman GH, et al. Comorbidity and functional status are independent in older cancer patients.J Clin Oncol 16: 1582-1587, 1998.

12. Repetto L, Fratino L, Audisio RA, et al.Comprehensive geriatric assessment adds information to Eastern Cooperative Oncology Group performance status in elderly cancer patients: an Italian Group for Geriatric Oncology Study. J Clin Oncol 20: 494-502, 2002.

13. Mihmanli M, Gurbulak EK, Akgun IE, et al. Delaying surgery after neoadjuvant chemoradiotherapy improves prognosis of rectal cancer. World J Gastrointest Oncol 8: 695-706, 2016.

14. Tulchinsky $H$, Shmueli $E$, Figer $A$, et al. An interval of $>7$ weeks between neoadjuvant therapy and surgery improves pathologic complete response and disease-free survival in patients with locally advanced rectal cancer. Ann Surg Oncol 15: 2661-2667, 2008.

15. De Campos Lobat LF, Geisler DP, da Luz Moreira A, et al. Neoadjuvant therapy for rectal cancer: the impact of longer interval between chemoradiation and surgery. J Gastrointest Surg 15: 444-450, 2011.

16. Mermut O, Inanc B, Arslan E, et al. Delayed Surgical Resection After Long-course Neoadjuvant Chemoradiotherapy in Rectal Cancer: Single Center Experience. Istanbul Med J 20: 424-430, 2019.

17. Bujko K, Nowacki MP, Nasierowska-Guttmejer A, et al. Longterm results of a randomized trial comparing preoperative short-course radiotherapy with preoperative conventionally fractionated chemoradiation for rectal cancer. Br J Surg 93: 1215-1223, 2006. 
International Journal of Hematology and Oncology

18. Ngan SY, Burmeister B, Fisher RJ, et al. Randomized trial of short-course radiotherapy versus long-course chemoradiation comparing rates of local recurrence in patients with T3 rectal cancer: Trans-Tasman Radiation Oncology Group trial 01.04. J Clin Oncol 30: 3827-3833, 2012.

19. Park SH, Kim JC. Preoperative chemoradiation for locally advanced rectal cancer: comparison of three radiation dose and fractionation schedules. Radiat Oncol J 34: 96-105, 2016.

20. Papamicheal D, Audisio RA, Glimelius B, et al.Treatment of colorectal cancer in older patients:International Society of Geriatric Oncology (SIOG) consensus recommendations 2013.Ann Oncol 26: 463-476, 2015.

21. Sun Q, Liu T, Liu P, et al.Adjuvant chemotherapy for locally advanced rectal cancer in elderly patients after neoadjuvant chemoradiotherapy and surgery: Toxicity and survival outcomes. Medicine (Baltimore) 99 (4): e18835, 2020.

22. Cirocco WC. Outcomes of rectal recestion following neoadjuvant therapy in the elderly:Can rectal cancer patients too old for a neoadjuvant approach? Am J Surg 215: 436-439, 2018.

23. Margalit DN, Mamon HJ, Ancukiewicz M, et al.Tolerability of combined modality therapy for rectal cancer in elderly patients aged 75 years and older.Int J Radiat Oncol Biol Phys 81: 735-741, 2011.

\section{Correspondence:}

\section{Dr. Berrin INANC}

Saglik Bilimleri Universitesi

Egitim ve Arastirma Hastanesi

Radyasyon Onkolojisi Anabilim Dali

34098, Fatih

ISTANBUL / TURKEY

Tel: (+90-212) 4596692

e-mail: byalcin77@hotmail.com

\section{ORCIDs:}

Berrin Inanc

0000-0002-6354-4609

Ozlem Mermut

0000-0002-5449-7361 